\title{
Preface to FP-UML 2011
}

The Unified Modeling Language (UML) has been widely accepted as the standard object-oriented language for modeling various aspects of software and information systems. The UML is an extensible language in the sense that it provides mechanisms to introduce new elements for specific domains; these include business modeling, database applications, data warehouses, software development processes, and web applications. Also, UML provides different diagrams for modeling different aspects of a software system. However, in most cases, not all of them need to be applied. Further, UML has grown more complex over the years, and new approaches are needed to effectively deal with these complexities. In general, we need heuristics and design guidelines that drive the effective use of UML in systems modeling and development.

The Seventh International Workshop on Foundations and Practices of UML (FPUML'11) will be a sequel to the successful BP-UML'05 - FP-UML'10 workshops held in conjunction with the ER'05 - ER'10 conferences, respectively. The FP-UML workshops are a premier forum for researchers and practitioners around the world to exchange ideas on the best practices for using UML in modeling and systems development. For FP-UML'11, we received papers from nine countries: Poland, Bosnia and Herzegovina, France, Germany, Israel, Mexico, Spain, Tunisia, and United States. While the papers addressed a wide range of issues, the dominant topic was model-driven architectures, including the various challenges related to transformations and the complexities resulting from multi-model specifications.

The Program Committee selected three papers to include in the program. The first paper by Brdjanin and Maric shows how associations in class diagrams can be generated from activity diagrams. The second paper by Reinhartz-Berger and Tsoury compares two core asset modeling methods, Cardinality-Based Feature Modeling and Application-Based Domain Modeling, and discusses their benefits and limitations in terms of specification and utilization capabilities. Finally, the third paper by Marth and Ren introduces the Actor-eUML model for concurrent programming and formalizes the mapping between actors in the Actor model and Executable UML agents by unifying the semantics of actor behavior and the hierarchical state machine semantics of Executable UML agents.

We thank the authors for submitting their papers, the program committee members for their hard work in reviewing papers, and the ER 2011 organizing committee for all their support.

July 2011

Guido L. Geerts Matti Rossi 